\title{
ADVANCED SURFACE FOR CONTROLLED OPTICAL PROPERTIES
}

\author{
${ }^{1}$ Peter BOHÁČIK, ${ }^{2}$ Jiří BÁRTA \\ 1VZLU - Czech Aerospace Research Centre, Prague, Czech Republic, EU, bohacik@vzlu.cz \\ ${ }^{2}$ VZLU -Czech Aerospace Research Centre, Prague, Czech Republic, EU, jiri.barta@vzlu.cz
}

https://doi.org/10.37904/metal.2020.3564

\begin{abstract}
This paper reports on anti-reflective (ARCs) and UV rejection coatings on variety of surfaces. The antireflective coatings have evolved into highly effective reflectance and glare reducing surfaces. The hindered amine light stabilizers (HALS) are very effective inhibitors against free radical included degradation of polymers at low and medium temperatures. HALS are based on 2,2,6,6,-tetramethyl-piperidine derivates. HALS can be categorized according their molecular weight, with low molecular weight (MW) about 200 to $500 \mathrm{~g} / \mathrm{mol}$ (low MW HALS) and those with molecular weight of 2000 or higher as high MW HALS. These classes have different rates of diffusion in the polymer matrix, this is important factor in protecting polymers from UV radiation. Very low HALS don't provide much thermal stability. Others like benzotriazoles and benzophenones are cost effective and provide better performance than other types of UV stabilizers. Benzotriazoles are widely used in high temperature resins. The antireflection coatings have usually two and more layers, but there are a single layer ARC. The single layer ARCs are based on silica nanoparticles with polymer substrate. A broad-spectrum light absorbing medium could be coated by $\mathrm{SiO}_{2}, \mathrm{SiO}, \mathrm{TiO}_{2}, \mathrm{ZrO}_{2}, \mathrm{Ta}_{2} \mathrm{O}_{5}$ or $\mathrm{Si}_{3} \mathrm{O}_{4}$ or by spin coating method. One of the layers could be an electrically conductive metal oxide, UV-stabilized polymers or material with lowrefractive index. These layers can be laminated. The aim of this study is to find a pathway for structure with low volatile organic compound (VOC) coating and second goal is to formulate novel UV rejection ARC.
\end{abstract}

Keywords: Surface, anti-reflectivity coating, UV rejection

\section{INTRODUCTION}

Optical coatings have been used in discovery, exploratory and monitoring missions since the beginning of space borne missions. The first application was in the 1958 at the Vanguard satellite in reaction to the USSR Sputnik a year earlier. Coatings that perform critical optical functions have been used in space instruments for the NASA. Pre-flight testing on the earth's surface in simulated space environments revealed changes in spectral and efficiency performance that are comparable to those changes observed in space. It has been learned that coating layers tend to absorb water in the atmosphere, and when inserted in the vacuum of space, the volatile water leaves. The coatings have evolved into e.g. anticorrosion, interior, frontage, impregnation or highly effective reflectance and glare reducing paints and coatings. Proper paint features can be achieved by optimization of paint mass formulation. Paint surface properties can be modelled by volume concentration of pigment (VCP) and by incorporation of additive such as polishers, defoamers etc. Paints VCP are divided in two groups: smooth and fine surface creating with low VCP and rougher surface creating with high VCP.

The coatings contain macromolecular binders which degrade under UV light. Therefore, it is necessary to protect them. One of the possibilities how to protect them is to use UV stabilizers. The stabilizers work on principle that is based on catching free radicals in a coat to acquire a stable non-reactive radicals, in the next step it is a reaction with another radical to acquire a "new" polymer chain. The UV stabilizer is ready to catch a next radical in the coating film. [1] The aim of this study is to find a pathway for structure with low volatile organic compound (VOC) coating and second goal is to formulate novel UV rejection ARC for use in cosmic environment. 


\section{ARC COATINGS}

Volatile organic compounds (VOC) are complication for coatings in space environment. If prepared coatings still contain VOC, after exposition to ultra-high vacuum, the residual solvents evaporate and destroy coating film and potentially blind sensors. Therefore, it is appropriate to use coatings with extremely low VOC or powder coatings. In real use, multi-layer ACR instead of single layer ACR are usually applied

For the antireflection coating, a reflection-reducing interference layer system containing a plurality alternating layer composed of high refractive index and low refractive index materials are usually used. These layers can be composed of poly-methyl methacrylate (PMMA) or other thermoplastics. [7] Most desirable approach is a broadband anti-reflection coating which is independent of the light incident angle. These coatings are nanostructures prepared e.g. on glass, quartz or other surface types by plasma etching method. The other possibility is an organic material combined in organic-inorganic hybrid layers applied to the alternating layers. This layer system has particularly low reflection over a wide wavelength range, This is based on the fact that the nanostructured layer composed of the organic or organic-inorganic hybrid materials have a porous structure with very low effective refractive index that could not be obtained with conventional materials for interference layer system. The thickness of nanostructured layers ranges from 50 to $150 \mathrm{~nm}$. Figure 1 shows the multi-layer ACR, which have 4 different types of interlayers and rough surface. [2-3-4]

\begin{tabular}{l} 
Layer 4 \\
\hline Layer 3 \\
\hline Layer 2 \\
\hline Layer 1 \\
\hline
\end{tabular}

Figure 1 Scheme of multi-layer ACR with rough surface, where for example layer 1 could be base - PMMA, layer 2 base protection - enamel, layer 3 thiouretane coating and layer 4 polycarbonate coating. Rough surface could be inorganic graphene coating

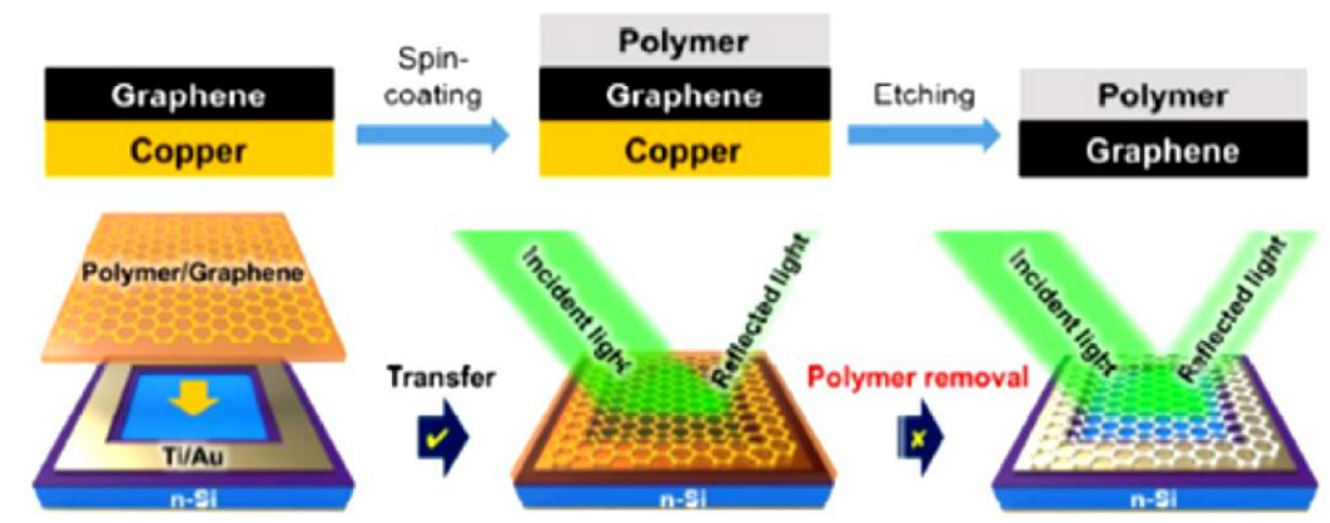

Figure 2 Various types of anti-reflective coatings (ARCs) based on the layer composition surface [6]

The polymer layer can be prepared by plasma polymerization. This type of polymerization of vaporous organic precursor compounds are firstly activated by plasma in a vacuum chamber. The result of activation is ionized 
molecules and these molecules undergo fragmentation creating clusters or chains. The condensation of these fragments on the surface brigs the polymer layer. Prepared polymer layers usually contain following compounds: styrene, allylamine, vinyl acetate, vinyl alcohol, hexamethylenedisoloxane (HMDSO), silicone, polyurethane, PMMA or melamine. The inter layers could be homo and copolymers or carbonates, diethylene glycol bisallylcarbonate, urethane, thiouretane, epoxide and combination of them. The useful materials for substrates are quartz, polyurethanes, polythiouretanes, acrylic/methacrylic/thioacrylic and polycarbonate polymers. Figure 2 shows types of ACR. [4-5-6]

\subsection{Single Layer ARC}

The Fresnel equation can be used to calculate the intensity of light reflecting on each surface. Here, $n_{s}$ represents a non-absorbing material through the refractive index at an ideal wavelength. In this regard, to decrease the substrate reflection down to zero at that wavelength the coating thickness should achieve the subsequent circumstances 1 and 2 .

$n_{\text {arc }}=\sqrt{n_{s} n_{\text {env }}}$

$d_{\operatorname{arc}} \frac{\lambda}{4 * n_{\operatorname{arc}}}$

where:
$n_{s}$ - represents a non-absorbing material through the refractive index at an ideal wavelength
$n_{\text {env }}$ - is the refractive index $(\mathrm{Rl})$ of the medium layer
$n_{\text {arc }}$ - is the refractive index $(\mathrm{RI})$ of the ARC surrounding respectively
$d_{\text {arc }}$ - is the ARC thickness

It should be noted that these equations are valid only for a nonadsorbing, homogenous medium, the loss of each medium will make the calculation more complicated. The biggest challenge for single layer with quarterwavelength ARCs is the decrease in the reflectivity for partial wavelengths and incidence angles, where they will vanish at glazing incidence angles. This is because of the varying incident light optical path-lengths of the glazing incidence. In this light, the phase difference between the incident and reflected waved cancel each other out when the glazing incidence is dependent on the norm.

In recent years, there have been several successful applications of $\mathrm{TiO}_{2}$ nano structures-based ARC on solar cells. One of the best candidates for single layer ARCs is inorganic coating similar to polymers. Even though they demonstrate lower refractive indices in comparison to inorganic ARCs, polymer coatings are stretchable, lightweight, and bendable. There are some researchers who describes use of such polymer on a substrate like graphene. An example is a study that applied PMMA as a single layer ARC on a layer of graphene to decrease the reflection. The fabrication process of this coating is simple, the results are considerable. The graphene was spin coated with PMMA ( $M_{w} 50,000,4$ wt.\% in ethyl lactate) at a speed (1,000-3,000 rpm). The copper substrate underneath the graphene film was etched away from the $\mathrm{FeCl}_{3} / \mathrm{HCl}$ solution, resulting in the PMMA coated graphene floating on the water surface.

The creation of suitable profiles could improve AR properties. The rendering of antireflective substrates has been demonstrated since the 1960s through surface texturing. The texture surface with wide angle shows the Figure 3. [6,7]

This study shows following, the module surface texturing and that the addition of an anti-reflective coating increases the clean photovoltaic module power out-put with a mean of between $4 \%$ and $8 \%$. 


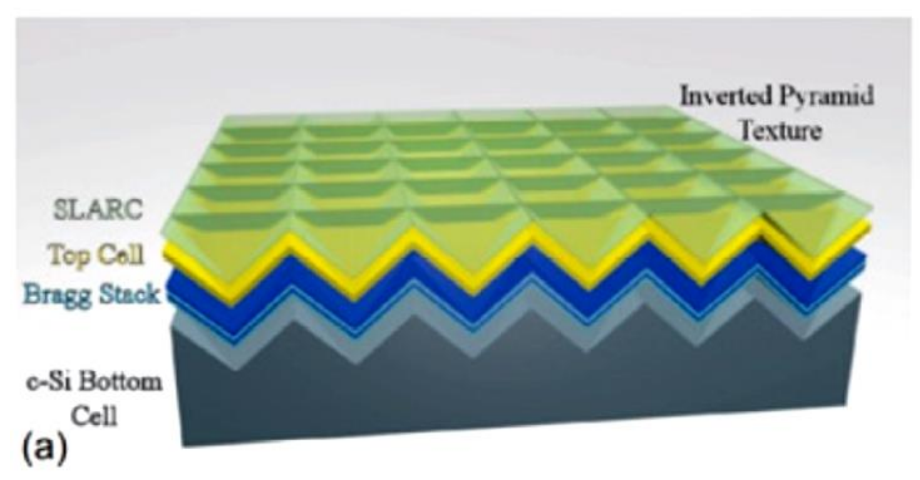

Figure 3 Textured surface wide angle view which shows the cross-sectional conformal deposition of the layers [6]

\section{CONCLUSION}

Presented article demonstrates the possibility to realize single layer $\mathrm{ARC}$ with $\mathrm{SiO}_{2}$ and $\mathrm{TiO}_{2}$. The multi layers are better than single-layer ARC, because there is a possibility to combine more layers for better radiation and UV rejection. As for UV rejection properties, there are several different types of HALS used. The structure polymers show better properties than non-structure polymers, that is why they have been chosen as a primary solution. For basic characterization, size exclusion chromatography (SEC) is used. For complete characterization and determination of structure, combination of SEC with multi angle scattering (MALS) and on-line viscosimeter is applied. The prepared structure polymers are used for formulation of VOC UV rejecting coatings for space environment.

\section{ACKNOWLEDGEMENTS}

This result was achieveded with the help of institutional funding of the Czech Ministry of Industry and Trade in the programme dedicated to the development of research organizations.

\section{REFERENCES}

[1] STEP, E.N., TURRO, N.J. Mechanics of polymer stabilization by Hindered-amine light stabilizers (HALS). Model investigation of the interaction of peroxy radicals with HALS amines and amino ethers. Macromolecules.1994, vol. 27, pp. 2525-2529.

[2] SCHULZ et al. Reflection-Reducing Interference Layer System and Method for Producing it., Germany., B2 US 8,192,639

[3] KAISER et al. Optical element with anti-fogging polymer layer, for use e.g. as spectacle lenses or display covers, has a reclection reducing nano-structure formed on the surface of the polymer layer., Germany., A1

DE 102,007,009,512

[4] THOMAS et al. Optical Article Covered with a Visible-Absorbing, Multi-Layer Anti-Reflective coating, and Production Method Thereof., France., B2 US 7,736,742

[5] MOAYEDFAR, M., ASSADI, K.M. Various types of anti-reflective coatings (ARCS) based on the layer composition and surface topography: A review. Reviews on Advanced Materials Science. 2018, vol. 53, pp. 187-205.

[6] GAN, X., ZHU, H., LV, R., MA, L.P., WANG, X., ZHANG, Z. Polymer coated graphene films as anti-reflective transparent electrodes for Schottky junction solar cells. Journal of Materials Chemistry A. 2016, vol. 4, pp. 13795-13802.

[7] SUltanOVA, N., KASAROVA, S., NIKOLOV, I. Dispersion Properties of Optical Polymers. Acta Physica Polonica-Series A General. 2009, vol. 116, pp. 585-588. 